\title{
Performance of Multi-Channel DBP with Long-haul Frequency-Referenced Transmission
}

\author{
Porto da Silva, Edson; Da Ros, Francesco; Zibar, Darko
}

Published in:

Proceedings of Optical Fiber Communications Conference and Exposition

Link to article, DOI:

10.1364/OFC.2016.Th2A.32

Publication date:

2016

Document Version

Peer reviewed version

Link back to DTU Orbit

Citation (APA):

Porto da Silva, E., Da Ros, F., \& Zibar, D. (2016). Performance of Multi-Channel DBP with Long-haul Frequency-Referenced Transmission. In Proceedings of Optical Fiber Communications Conference and Exposition [7537369] Optical Society of America (OSA). https://doi.org/10.1364/OFC.2016.Th2A.32

\section{General rights}

Copyright and moral rights for the publications made accessible in the public portal are retained by the authors and/or other copyright owners and it is a condition of accessing publications that users recognise and abide by the legal requirements associated with these rights.

- Users may download and print one copy of any publication from the public portal for the purpose of private study or research.

- You may not further distribute the material or use it for any profit-making activity or commercial gain

- You may freely distribute the URL identifying the publication in the public portal 


\title{
Performance of Multi-Channel DBP with Long-haul Frequency-Referenced Transmission
}

\author{
Edson Porto da Silva*, Francesco da Ros, and Darko Zibar \\ DTU Fotonik, Technical University of Denmark, DTU, Kongens Lyngby, 2800, Denmark, DK \\ *edpod@fotonik.dtu.dk
}

\begin{abstract}
The impact of frequency referenced WDM source on the performance of dual polarization multi-channel digital backpropagation (MC-DBP) is experimentally investigated up to $4000 \mathrm{~km}$ of transmission. For a system with $4 \times 8 \mathrm{GBd}$ DP-QPSK, such approach allows $0.6 \mathrm{~dB}$ more MC-DBP $Q^{2}$-factor gain in the nonlinear regime, compared with an unreferenced scheme.
\end{abstract}

OCIS codes: (060.1660) Coherent communications, (060.2360) Fiber optics links and subsystems

\section{Introduction}

In order to compensate for deterministic inter-channel nonlinear distortions, the receiver or the transmitter must be able to jointly process all carriers which are co-propagating within the fiber. For the cases where this condition is fulfilled, inter-carrier nonlinearity compensation (NLC) schemes can be applied. Recently, multi-channel digital backpropagation (MC-DBP) approaches have been numerically [1] and experimentally [2] investigated to compensate both intra and inter-carrier nonlinear effects. However, as first pointed out in [3], since multi-channel NLC techniques rely on approximate solutions of the nonlinear Schrödinger equation (NLSE), a fundamental limitation to their effectiveness is imposed by inherent uncertainty in the frequency spacing between co-propagating carriers in a wavelength division multiplexing (WDM) system. To tackle this problem, frequency-referenced transmission was proposed in [3]. By this approach, all transmitted carriers are generated from a stable optical frequency comb, in order to conserve a locked frequency separation. Such strategy is proved to benefit multi-channel NLC performed at the transmitter, with the use of nonlinear pre-distortion [3,4]. Previous works have presented experimental investigations on the performance of multi-channel nonlinear pre-distortion for frequency locked and frequency unlocked carrier transmission [5-7]. However, no investigation is provided regarding the impact of such scheme on the performance of full-field MC-DBP on the receiver side. Additionally, [5-7] investigate frequency-referenced transmission only for single-polarization WDM systems.

Full-field MC-DBP has a lower implementation cost than nonlinear pre-distortion, since the last one implies extra requirements on synchronization, bandwidth, and resolution of digital-to-analog converters (DACs) at the transmitters. More importantly, different from pre-distortion, full-field MC-DBP based on Split-Step Fourier Method (SSFM) does not assume any previous carrier frequency allocation in order to perform the inverse channel propagation. Therefore, the use of frequency-referenced transmission may result in distinct performance impacts, if NLC is performed using receiver-side MC-DBP instead of nonlinear pre-distortion at the transmitter. In [2] the performance of $\mathrm{MC}$-DBP is evaluated in a scheme with locked carriers transmission. However, no comparison to the case of unlocked carriers transmission is provided.

In this paper, we present the first experimental investigation on the impact of frequency-referenced long haul transmission on the performance of receiver-side full-field MC-DBP for polarization multiplexed systems. We evaluate the MC-DBP performance of a $4 \times 8 \mathrm{GBd}$ dual-polarization quadrature phase-shift keying (DP-QPSK) system, with and without the use of frequency-referenced carriers, up to $4000 \mathrm{~km}$ of reach.

\section{Experimental setup}

The experimental setup is shown in Fig. 1. At the transmitter side, two in-phase and quadrature (IQ) modulators are used to modulate two independent external cavity lasers, both with $100 \mathrm{kHz}$ linewidth. The lasers' central frequencies are separated by $8.5 \mathrm{GHz}$. The RF signals used to drive the modulators are provided by an arbitrary waveform generator (AWG) with a sample-rate of $64 \mathrm{GSa} / \mathrm{s}$ and analog bandwidth of $20 \mathrm{GHz}$. The four channels of the AWG are amplified to $5.0 \mathrm{~V}$ of peak-to-peak amplitude to drive the modulators. Offline digital signal processing (DSP) is used to pre-compensate the combined frequency response of the AWG channels, RF cables, and amplifiers. The modulated baseband signal synthesized by the AWG is generated in two different subcarrier configurations: in the case 1, only 


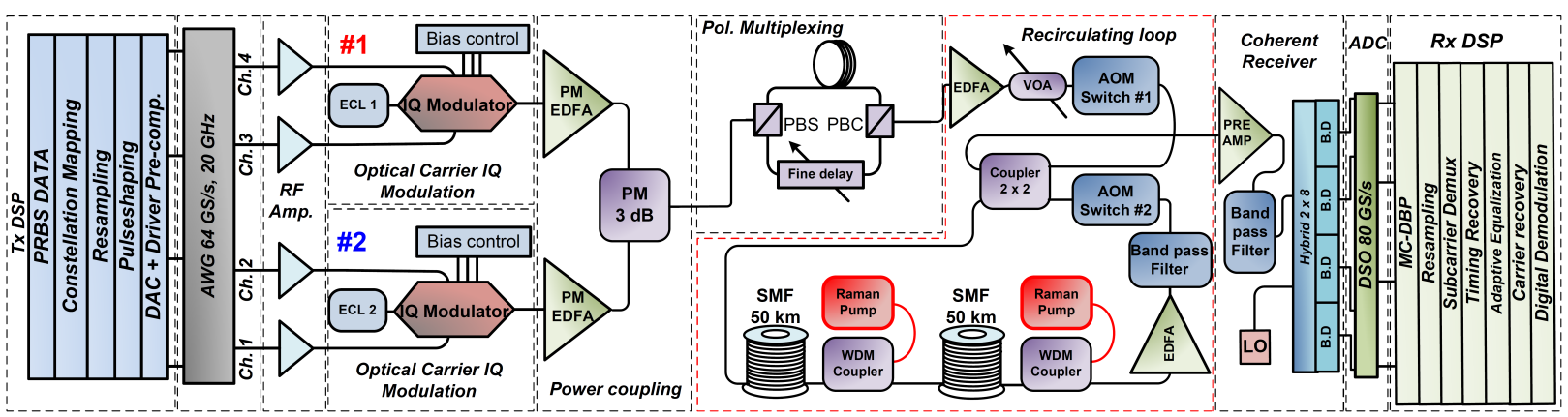

Fig. 1: Experimental setup used to evaluate the performance of receiver-side MC-DBP in frequency-referenced polarization multiplexed long-haul transmission.

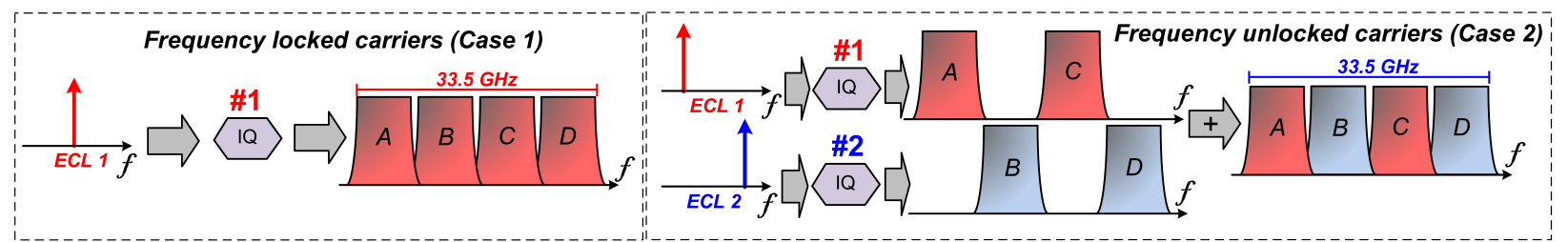

Fig. 2: Schematic of the two configurations used to generate the transmitted carriers. (a) Only one laser is modulated. Four decorrelated carriers spaced of $8.5 \mathrm{GHz}$ are generated directly from the AWG output. (b) Two free-running lasers are modulated by different IQ modulators. The AWG output generates two carriers spaced of $17 \mathrm{GHz}$. Four decorrelated carriers spaced of $8.5 \mathrm{GHz}$ are obtained after $3 \mathrm{~dB}$ coupling, with even and odd originated from different lasers.

modulator \#1 in Fig. 1 is driven. The two IQ AWG channels connected to \#1 generate four decorrelated QPSK carriers, each modulated at $8 \mathrm{GBd}$, with root raised cosine (RRC) pulse shape with a roll-off factor of $1 \%$, and separated by $500 \mathrm{MHz}$ (6.25\% of the baud rate) guard bands, as shown in Fig. 2 (a). The output of modulator \#1 is followed by polarization maintaining (PM) erbium doped fiber amplifier (EDFA). In the configuration of case 1, modulator \#1 outputs a superchannel with four frequency locked carriers. For case 2, modulators \#1 and \#2 in Fig. 1 are driven simultaneously. The AWG sends two decorrelated QPSK carriers to each modulator, each carrier modulated at $8 \mathrm{GBd}$, with RRC pulse shape (roll-off factor of $1 \%$ ), and separated of $17 \mathrm{GHz}$, as shown in Fig. 2 (b). In the configuration of case 2, the outputs of the modulators are amplified by PM EDFAs and combined with a $3 \mathrm{~dB}$ PM coupler, in order to generate a superchannel with the same characteristics of case 1, but with even and odd carriers generated by two independent free running lasers. Although for case 2 not all carriers are unlocked in frequency, such configuration is valid for test case comparison, according with what is presented in [3].

In both cases, the data of all QPSK carriers is mapped from a pseudo random bit sequence (PRBS, $\left.2^{23}-1\right)$. The sequence of independently modulated carrier allocation $(A, B, C, D)$ was intentionally chosen to avoid possible inconsistency on the results obtained in the nonlinear transmission regime due to correlation imposed by even and odd $(A, B, A, B)$ data allocation [9]. After the power coupling stage in Fig. 1, the modulated carriers are then sent to a polarization multiplexing emulation stage, in order to get the final DP-QPSK desired superchannel. The multi-carrier signal is then amplified by a booster EDFA, sent to a variable optical attenuator and connected to the input of a recirculating loop. The passive structure of the loop is composed by two $50 \mathrm{~km}$ spans of standard single mode fiber (SMF), an optical bandpass filter, a 2-by-2 coupler $(3 \mathrm{~dB})$ and two acousto-optic switches. All fiber losses are compensated by Raman amplification, performed with a backward pumping scheme per span (26 dBm@1450 nm). An EDFA is positioned inside the loop to compensate for the remaining losses. At the output of the recirculating loop, the signal is pre-amplified, filtered to suppress out of band noise, and coherently detected with a single optical coherent receiver. The detected signal is sampled and acquired with a real time sampling oscilloscope at $80 \mathrm{GSa} / \mathrm{s}$ and $33 \mathrm{GHz}$ of bandwidth per acquisition channel. We then use offline DSP [8] to demodulate the received carriers. The flow of DSP algorithms is composed by full-field MC-DBP, resampling, subcarrier demultiplexing (downconversion and matched filtering), timing recovery, adaptive equalization with the constant modulus algorithm (CMA), carrier recovery, digital demodulation, and bit error counting. Full-field DBP is performed using a non-iterative symmetric SSFM to solve the Manakov approximation of the NLSE, with a fixed step size of $0.625 \mathrm{~km}$ ( 80 steps per $50 \mathrm{~km}$ span). For both test cases, the transmitted signal was acquired after propagation over nine distinct transmission distances $(L)$, up to $4000 \mathrm{~km}$, with the total fiber launch power $\left(P_{i n}\right)$ varied from $-6 \mathrm{dBm}$ to $6 \mathrm{dBm}$ (i.e. $-12 \mathrm{dBm}$ to $0 \mathrm{dBm}$, per carrier) in steps of $1 \mathrm{~dB}$, providing a total of 117 datasets, corresponding to the number of tested $\left(L, P_{i n}\right)$ configurations. Each processed dataset had $10^{6}$ signal samples, which allowed a minimum reliable bit error rate (BER) counting of $10^{-4}$ 


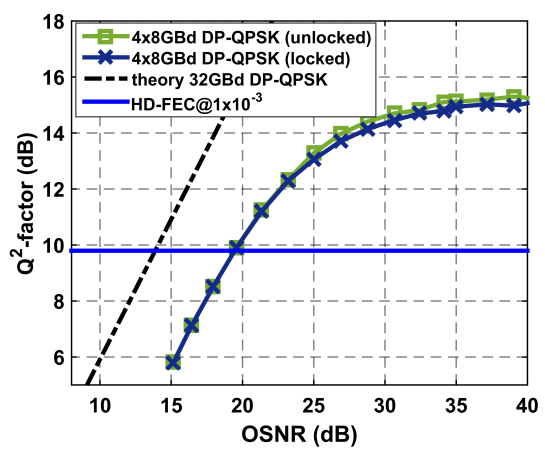

(a) Back-to-back performance.

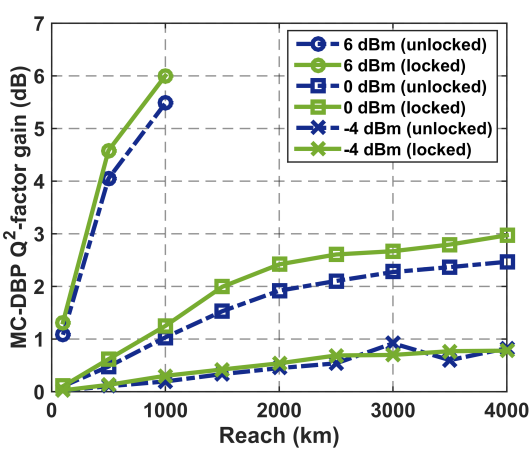

(b) MC-DBP performance gain.

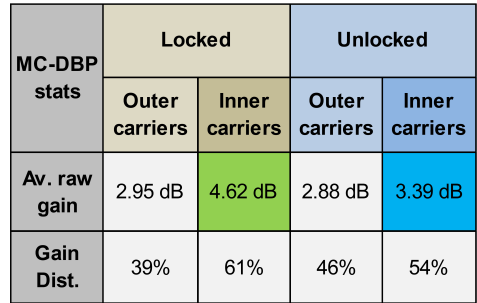

(c) Average MC-DBP gain distribution per carriers

Fig. 3: Experimental results. (a) Back-to-back characterization for case 1 and case 2. (b) Obtainable gain of MC-DBP as a function of transmission distance for frequency locked and unlocked carriers, for the input powers of: $-4 \mathrm{dBm}$ (optimal launch power with EDC only), $0 \mathrm{dBm}$ and $6 \mathrm{dBm}$ (nonlinear regime). (c) Summary of average gain and gain distribution for all processed datasets $\left(L, P_{i n}\right)$.

per carrier (i.e, $Q^{2}$-factor of $11.4 \mathrm{~dB}$ ), before hard-decision forward error correction (pre-HD-FEC).

\section{Results and discussion}

In Fig. $3 a$ and Fig. 3b, the $Q^{2}$-factor is calculated from the average BER of all carriers $\left(Q^{2}=\right.$ $\left.20 \log _{10}\left[\sqrt{2} \operatorname{erfcinv}\left(2 . B E R_{a v}\right)\right]\right)$ is used here as figure of merit for performance. For the error free data sets, the $Q^{2}$ factor was estimated using the error vector magnitude (EVM) calculated from the error free received constellation (i.e. $Q^{2}$-factor values higher than $11.4 \mathrm{~dB}$ ). In Fig. 3a the back-to-back performance in $Q^{2}$-factor as function of OSNR for the $4 \times 8$ GBd DP-QPSK superchannel is depicted for the two tested configurations. Similar performance is obtained for frequency locked (case 1) and frequency unlocked (case 2) carriers transmitters. An implementation penalty of $5 \mathrm{~dB}$ compared with the theoretical curve is noticed. This is mainly due to the impact of peak-to-average power ratio (PAPR) of the baseband signal [10]. Two factors contribute to increase the PAPR of the signal delivered by the AWG: multicarrier generation and low roll-off RRC filtering. However, since both cases presented negligible differences in the back-to-back characterization, the performance after transmission can be compared in a common basis.

In Fig. $3 b$ the performance of MC-DBP is quantified and compared for both tested cases, at different transmission distances. The gain is calculated with respect to the performance obtained using only frequency domain electronic compensation of chromatic dispersion (EDC). The curves shown correspond to three fiber total input power values: $-4 \mathrm{dBm}$ (optimum launch power with EDC only), $0 \mathrm{dBm}$ and $6 \mathrm{dBm}$. At $-4 \mathrm{dBm}$ of total launch power (i.e. $-10 \mathrm{dBm}$ per carrier), the MC-DBP gain is basically the same for both cases and all distances. However, for launch power values in the nonlinear transmission regime, $0 \mathrm{dBm}$ and $6 \mathrm{dBm}$, the MC-DBP gain of the locked carriers configuration tends to be $0.6 \mathrm{~dB}$ better than the gain for the unlocked case for long haul distances $(>1000 \mathrm{~km})$. In Fig. $3 \mathrm{c}$ the $Q^{2}$-factor gain averaged over all $\left(L, P_{i n}\right)$ acquired datasets is shown for each test case. It is noticed that the average $Q^{2}$-factor gain for the locked configuration is $1.3 \mathrm{~dB}$ higher than the one obtained for the unlocked case for the inner carriers $(\mathrm{B}$ and $\mathrm{C}$, in Fig. 2). The average $Q^{2}$-factor gain observed for the outer carriers (A and D, in Fig. 2) was approximately the same in both configurations. Therefore, one can conclude that frequency-referenced transmission also helps the performance of full-field MC-DBP. However, such performance advantage was only identified in the nonlinear transmission regime, for launch power values higher than the optimum.

\section{Conclusion}

The results show that the use of frequency-referenced transmission has influence on receiver-side full-field MC-DBP performance. In the performed $4 \times 8 \mathrm{GBd}$ DP-QPSK long-haul transmission experiment, the use of a frequencyreferenced scheme has provided an average improvement of $0.6 \mathrm{~dB}$ in the $Q^{2}$-factor gain obtained by MC-DBP in the nonlinear regime, with respect to the gain obtained when the backpropagated carriers are not frequency locked.

\section{References}

[1] G. Liga, et al, Opt. Express , 22 (24), Nov. (2014).

[2] R. Maher, et al, Sci. Rep., vol. 5, Jan. (2015).

[3] N. Alic, et al, J. Light. Technol. , 32(15), Aug. (2014).

[4] R.-J. Essiambre, et al, IEEE Photon. Technol. Lett. , 18(17), Sep. (2006).

[5] E. Temprana, et al, Science, 348(6242), Jun. (2015).
[6] E. Temprana, et al, Opt. Express , 23(16), Aug. (2015).

[7] E. Temprana, et al, Proc. ECOC, paper Th.2.6.5 (2015).

[8] R. Borkowski, et al, IEICE Trans. on Comm., 97(8), (2014).

[9] L. B. Du and A. J. Lowery, Opt. Express , 20(26), Dec. (2012).

[10] S. H. Han and J. H. Lee, IEEE Wireless Commun., 12(2), Apr. (2005). 